\title{
Improvements of volume holographic grating in photopolymer doped with nanorods \\ (Conference Presentation) (Withdrawal Notice)
}

Liangcai Cao, Shenghan Wu, Song Zong, Hao Zhang, Guofan Jin

Liangcai Cao, Shenghan Wu, Song Zong, Hao Zhang, Guofan Jin, "Improvements of volume holographic grating in photopolymer doped with nanorods

(Conference Presentation) (Withdrawal Notice)," Proc. SPIE 9958, Photonic Fiber and Crystal Devices: Advances in Materials and Innovations in Device Applications X, 99580H (15 January 2018); doi: 10.1117/12.2238823

SPIE Event: SPIE Optical Engineering + Applications, 2016, San Diego, California, United States 


\section{Improvements of volume holographic grating in}

photopolymer doped with nanorods (Conference Presentation) (Withdrawal Notice)

Proc. SPIE 9958, 99580H (2016)

Online Publication Date: 2 November 2016

Withdrawn from Publication: 15 January 2018

Conference Date: 28-29 August 2016

Conference Location: San Diego, United States

Conference Title: Photonic Fiber and Crystal Devices: Advances in Materials and Innovations in Device Applications X Conference Chairs: Shizhuo Yin, Ruyan Guo

Liangcai Cao

Tsinghua Univ. (China)

Publisher's Note: This conference presentation, originally published on 2 November 2016, was withdrawn per author request. 